\title{
Zero-order filter for diffractive focusing of de Broglie matter waves
}

Eder, S. D. ; Ravn, A. K. ; Samelin, B. ; Bracco, G.; Salvador Palau, A. ; Reisinger, T.; Bergbäck Knudsen, Erik

Published in:

Physical Review A

Link to article, DOI:

10.1103/PhysRevA.95.023618

Publication date:

2017

Document Version

Publisher's PDF, also known as Version of record

Link back to DTU Orbit

Citation (APA):

Eder, S. D., Ravn, A. K., Samelin, B., Bracco, G., Salvador Palau, A., Reisinger, T., \& Bergbäck Knudsen, E. (2017). Zero-order filter for diffractive focusing of de Broglie matter waves. Physical Review A, 95, [023618]. https://doi.org/10.1103/PhysRevA.95.023618

\section{General rights}

Copyright and moral rights for the publications made accessible in the public portal are retained by the authors and/or other copyright owners and it is a condition of accessing publications that users recognise and abide by the legal requirements associated with these rights.

- Users may download and print one copy of any publication from the public portal for the purpose of private study or research.

- You may not further distribute the material or use it for any profit-making activity or commercial gain

- You may freely distribute the URL identifying the publication in the public portal 


\title{
Zero-order filter for diffractive focusing of de Broglie matter waves
}

\author{
S. D. Eder, ${ }^{1}$ A. K. Ravn,${ }^{2}$ B. Samelin, ${ }^{1}$ G. Bracco, ${ }^{3}$ A. Salvador Palau,,${ }^{1}$ T. Reisinger, ${ }^{1, *}$ \\ E. B. Knudsen, ${ }^{4}$ K. Lefmann, ${ }^{2}$ and B. Holst ${ }^{1, \dagger}$ \\ ${ }^{1}$ Department of Physics and Technology, University of Bergen, Allégaten 55, 5007 Bergen, Norway \\ ${ }^{2}$ Nanoscience Center, Niels Bohr Institute, University of Copenhagen, 2100 Copenhagen, Denmark \\ ${ }^{3}$ Department of Physics, IMEM, CNR, University of Genova, Via Dodecaneso 33, 16146 Genova, Italy \\ ${ }^{4}$ NEXMAP, Physics Department, Technical University of Denmark, 2800 Kongens Lyngby, Denmark
}

(Received 4 August 2016; published 21 February 2017)

\begin{abstract}
The manipulation of neutral atoms and molecules via their de Broglie wave properties, also referred to as de Broglie matter wave optics, is relevant for several fields ranging from fundamental quantum mechanics tests and quantum metrology to measurements of interaction potentials and new imaging techniques. However, there are several challenges. For example, for diffractive focusing elements, the zero-order beam provides a challenge because it decreases the signal contrast. Here we present the experimental realization of a zero-order filter, also referred to as an order-sorting aperture for de Broglie matter wave diffractive focusing elements. The zero-order filter makes it possible to measure even at low beam intensities. We present measurements of zero-order filtered, focused, neutral helium beams generated at source stagnation pressures between 11 and 81 bars. We show that for certain conditions the atom focusing at lower source stagnation pressures (broader velocity distributions) is better than what has previously been predicted. We present simulations with the software ray-tracing simulation package MCSTAS using a realistic helium source configuration, which gives very good agreement with our measurements.
\end{abstract}

DOI: 10.1103/PhysRevA.95.023618

\section{INTRODUCTION}

de Broglie matter wave optics has attracted much interest in recent years. For reviews related to quantum metrology and fundamental quantum mechanics tests, see [1-3]. de Broglie matter waves created by supersonic expansion, in particular neutral helium beams, are established tools in surface science [4,5]. Presently, helium beams are mainly used in studies of surface diffraction and dynamics, but the research is ongoing for applying them in microscopic imaging. The helium beam has a low energy (typically less than $100 \mathrm{meV}$ ) and does not penetrate solid material: The helium beam maps the electron density distribution of the surface. This makes neutral helium microscopy an attractive candidate for the imaging of insulating and/or fragile surface structures and nanocoatings. By using two detectors, recording the scattered beams at different angles, it should even be possible, in principle, to make a nanoscale stereo microscope. The first helium focusing experiments of neutral helium were carried out by Carnal et al. [6] using a metastable beam and later by Doak et al. [7] using a ground-state helium beam. The first neutral helium microscopy images were obtained using a diffractive focusing element: A Fresnel-Soret zone plate (zone plate with a square-wave amplitude transmission function) was used to focus a helium beam down to a few micrometers [8] and later even below $1 \mu \mathrm{m}$ [9]. An alternative pinhole helium microscopy setup was subsequently introduced [10-13]. The first reflection microscopy images were obtained with this method [10]. Diffractive focusing elements have also been used to focus molecules [14] and to measure atom interaction potentials

\footnotetext{
*Present address: Institute of Nanotechnology, Karlsruhe Institute of Technology, Hermann-von-Helmholtz-Platz 1, 76344 EggensteinLeopoldshafen, Germany.

†bodil.holst@uib.no
}

$[15,16]$ and have been proposed as a method for investigation of the Casimir-Polder force [17].

Because of the low energy of the atoms or molecules in the beams used in de Broglie matter wave optics experiments, the beams cannot penetrate solid material as mentioned above and therefore the diffracting focusing elements have to work by reflection or for transmission must be free-standing suspended structures. This is a fabrication challenge and limits the number of possible elements. Fresnel-Soret zone plate focusing has been used on several occasions as described above (see also $[7,18])$. Recently, another diffracting focusing element, the atom sieve, was introduced [19]. The atom sieve is based on the photon sieve invented earlier [20]. It consists of pinholes of varying size arranged across the Fresnel zones in such a manner that it is possible to focus on a spot with a diameter smaller than the smallest pinhole. In addition, higher-order diffraction and secondary maxima can be suppressed by several orders of magnitude.

As an alternative to Fresnel zone plates, mirrors can be used as focusing elements [21-23]. The use of graphene as a mirror coating gives an inert and very stable surface [24]. In addition, one-dimensional focusing with quantum reflection from a curved substrate has been demonstrated [25].

Up until now it has not been possible to control the curvature of mirrors with high precision, so Fresnel-zone-based optical elements remain the most promising approach for the focusing of neutral-atom and molecular beams, but the zero-order contribution is a major issue. For a standard Fresnel-Soret zone plate, $25 \%$ of the incident beam (half of the transmitted intensity) goes into the zero-order focus and only about $10 \%$ into the first-order focus [26]. The standard procedure, also adopted in x-ray applications, is to block the central part of the zone plate with an opaque middle stop, but this does not prevent the zero-order beam from contributing through the open fraction of the focusing element and the larger the middle stop, the higher the intensity loss. To filter out the last fraction of the zero 
order a so-called order-sorting aperture needs to be applied, as is done, for example, in nanoscale x-ray imaging [27].

In this paper we present the realization of an order-sorting aperture (zero-order filter) for de Broglie matter waves. An additional advantage apart from the zero-order filtering is that it is possible to reduce the size of the middle stop so that the intensity in the focus can be increased. This is particularly important for de Broglie matter waves, because they often have low intensity. The successful implementation of the zero-order filter has enabled us to test systematically the focus size of neutral helium beams at lower pressures and we present a Monte Carlo simulation model of the focusing for all pressure regimes based on the MCSTAS instrument simulation software for neutron instrumentation [28,29].

\section{EXPERIMENTAL SETUP}

The experiments presented here were carried out in the neutral helium microscope prototype instrument at the University of Bergen. The microscope is popularly referred to as NEMI, short for neutral microscope. We will adhere to this convention for the rest of the paper. The neutral helium beam was created by a free-jet expansion from a source reservoir through a $(5 \pm$ 1)- $\mu$ m-diam nozzle. The central part of the beam was selected by a skimmer placed $11.5 \pm 0.5 \mathrm{~mm}$ in front of the nozzle. Two different skimmers were used: a $(10 \pm 1)-\mu$ m-diam skimmer and a (50 \pm 2$)$ - $\mu \mathrm{m}$-diam skimmer. The beam source in NEMI has been specifically designed for microskimmer experiments, allowing one to position the skimmer relative to the nozzle with submicrometer precision [30].

A drawing of NEMI can be seen in Fig. 1. The beam transmitted through the microskimmer is focused by a FresnelSoret zone plate $192 \mu \mathrm{m}$ in diameter with a $50-\mu \mathrm{m}$-diam middle stop [31] onto a translation stage (PI miCos). For the experiments presented here, a $10-\mu \mathrm{m}$-wide slit was mounted on the translation stage and used to scan the beam. The beam fraction transmitted through the slit was detected in a pitot tube detector placed under the translation stage.

The Fresnel zone plate used for the experiments presented here is designed to have a focal length of $168.14 \mathrm{~mm}$ for a wavelength of $\lambda=0.055 \mathrm{~nm}$, corresponding to a beam temperature of about $320 \mathrm{~K}$ (there is a small wavelength dependence also with the source reservoir pressure). The focal length is chosen to fit the geometry of the NEMI system with an object distance $g$ (distance between the skimmer and Fresnel zone plate) of $935 \mathrm{~mm}$ and an image distance $b$ (distance between Fresnel zone plate and slit plane) of $205 \mathrm{~mm}$.

All experiments were carried out at source pressures between 11 and 81 bars. The corresponding most probable He beam wavelength varies slightly with pressure from $\lambda \approx$ $0.0555 \pm 0.0004 \mathrm{~nm}$ to $\lambda \approx 0.0564 \pm 0.0004 \mathrm{~nm}$. The width of the velocity distribution, and thereby the width of the wavelength distribution, varies much more. Traditionally, this beam property is quantified using the parameter of speed ratio $S$, where $S$ is defined as $S=2 \sqrt{\ln (2)} v / \Delta v$ [32], where $\Delta v$ is the full width at half maximum (FWHM) of the velocity distribution and $v$ the most probable beam velocity. Since NEMI does not have a time-of-flight system, theoretical values for the speed ratios were obtained by numerically solving the

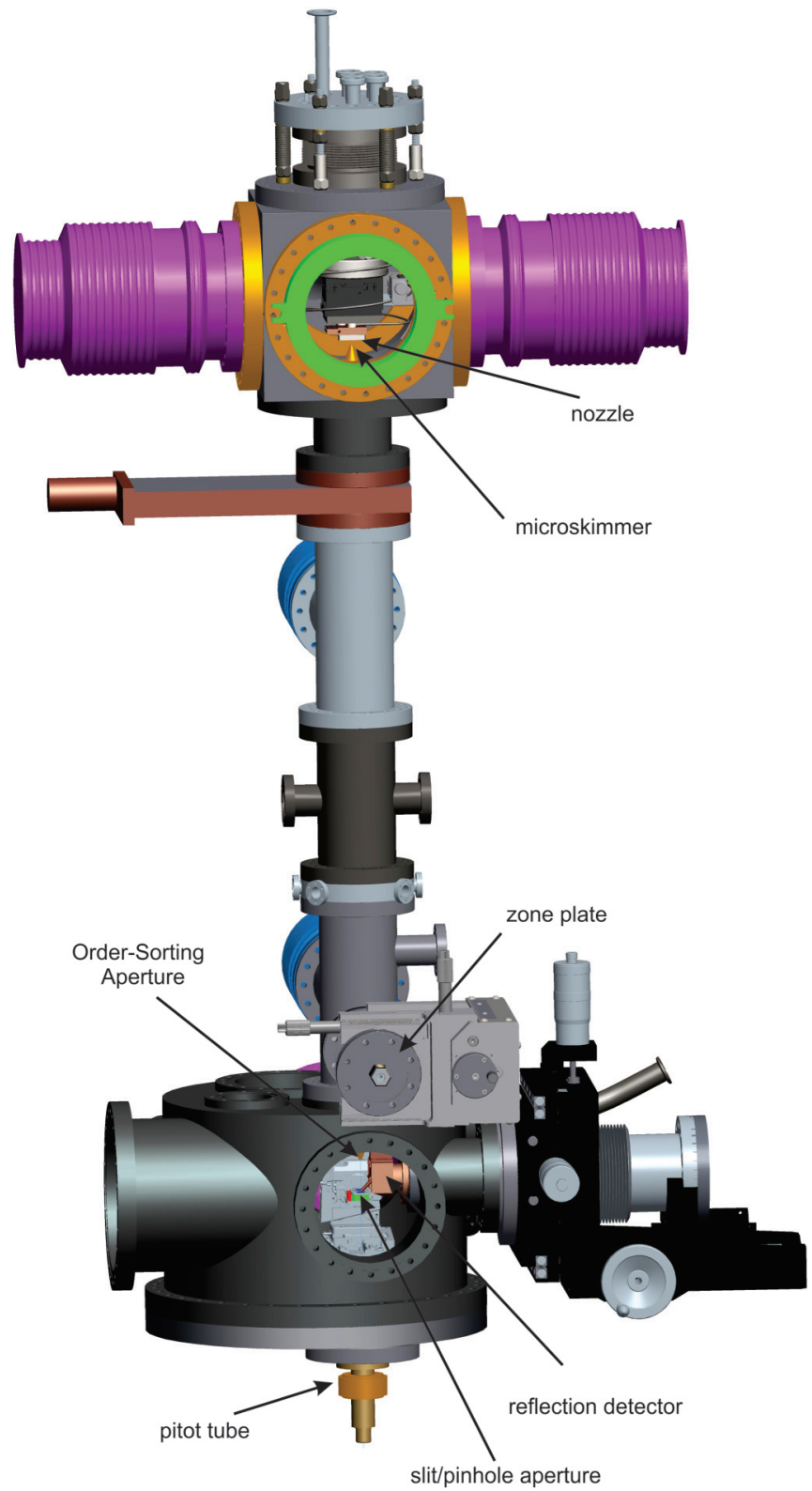

FIG. 1. Drawing of NEMI used to perform the measurements presented here. The focused helium atom spot profile is observed by moving a $10-\mu \mathrm{m}$ slit aperture, mounted on a translation stage, across the focused spot and measuring the pressure increase in the Pitot tube detector. To the right the detector setup used for reflection imaging can be seen. It is not used for the experiments presented here.

Boltzmann equation $[15,33,34]$. The speed ratio varied from 9.0 to 25.8 .

\section{THEORETICAL BACKGROUND}

\section{A. Analytical model}

The size of the focused helium atom spot is determined by the geometry of the system, the size of the source, and the chromatic aberration caused by the velocity spread of the beam. The chromatic aberration for a Fresnel zone plate can be described by the transversal width of the chromatic point spread function [35]. Using a simple geometrical argument 


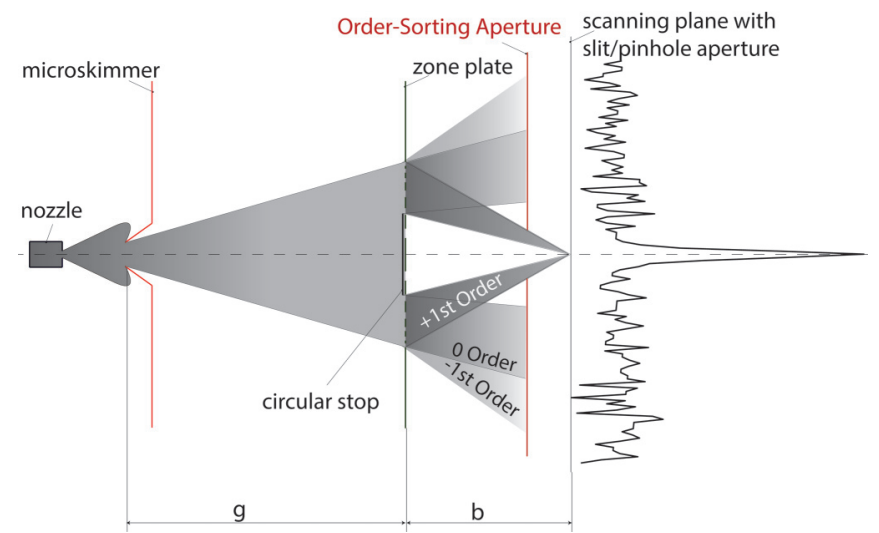

FIG. 2. Diagram of the zero-order filter setup. After the free-jet expansion through the nozzle, the central part of the helium beam is selected by the microskimmer. The beam hits the Fresnel zone plate. The central part of the zone plate has been blocked by a circular middle stop, which filters part of the zero-order beam. The rest of the zero-order beam (and most of the higher orders) is blocked by the order-sorting aperture with a diameter slightly smaller than the middle stop.

as described in [14], we get an expression for the transversal width $w$,

$$
w=\sqrt{\ln (2)} \frac{D_{\mathrm{ZP}}}{S},
$$

where $D_{\mathrm{ZP}}$ is the diameter of the Fresnel zone plate and $S$ is the speed ratio of the beam. In the model it is assumed that the point spread function does not change significantly when slightly off axis and hence that each point on the source will contribute with a point spread function of the same size. The final focused spot size $d_{\text {focus }}$ can thus be described as a convolution of the transversal width $w$ with the geometrical image of the source. A simplified model for the final focused spot size is given by approximating the source image and $w$ with Gaussian functions. The skimmer diameter $d_{\mathrm{sk}}$ is taken to be the FWHM of the object function [9]. We thus obtain for the theoretical final spot size $d_{\text {focus }}$,

$$
d_{\text {focus }}=\sqrt{w^{2}+\left(M d_{\mathrm{sk}}\right)^{2}},
$$

where $M=b / g$ is the magnification factor given by the geometry of the system (see Fig. 2).

\section{B. MCSTAS simulations}

MCSTAS [36] is a ray-tracing simulation software package developed specifically with the purpose of simulating neutron scattering instruments and experiments [28,29], distributed using the GNU General Public License. The domain specific language (DSL) is built on ANSI-C. There are three levels of coding in MCSTAS. The bottom level is the MCSTAS kernel, where all low-level particle transportation routines, geometry engines, etc., are placed. It is written in ANSI-C and provides the basis for the MCSTAS DSL and compiler used on the other levels. The middle level is the component files. These files are the building blocks of the MCSTAS simulated instruments and here the Monte Carlo choices are taken. The top level is the instrument file. It consists of a number of calls to different components and a geometrical description of the instrument setup.

The assumption used throughout the simulations is that the helium atoms behave just like neutrons, only four times heavier. As long as we are only interested in the diffraction properties and set the material parameters so that the "neutrons" cannot penetrate any material, this is a valid assumption. MCSTAS does not take into account interaction between particles, which is appropriate for the description of the supersonic expansion beam used here.

In order to simulate NEMI in MCSTAS, four instrument components were implemented: (i) a beam source, (ii) a zone plate, $192 \mu \mathrm{m}$ in diameter with a 50- $\mu \mathrm{m}$ middle stop, (iii) the corresponding zero-order filter with a $40-\mu$ m opening, and (iv) a detection plane. The beam source has been implemented as a ray-tracing version of the ellipsoidal quitting surface model with a $\cos ^{3}$ density distribution over the quitting surface as described in [37]. The quitting surface represents the distance from the nozzle in any direction, where the atoms have reached molecular flow and are no longer interacting. The velocity distribution over the quitting surface is obtained by solving numerically the Boltzmann equation [15,33,34] (see also Ref. [38]).

The simulations were carried out by tracing around $10^{8}$ rays, originating from the source. The starting position and velocity of each ray was selected at random, according to the source intensity and velocity distribution described above. MCSTAS in its present form does not include the phase in the simulations. Each ray was traced to the plane of the zone plate. Using the velocity (wavelength) associated with the ray, the standard formula for the wavelength-dependent focal length of a zone plate [35], the starting position of the ray at the quitting surface, and the position of the ray at the zone plate, the position of the ray in the image plane was determined. This position was then traced back or forward to the detector plane. To simulate the experiment, the rays were binned in $10-\mu \mathrm{m}$-wide slots at $0.3-\mu \mathrm{m}$ distance, which corresponds to the readout positions of the $10-\mu \mathrm{m}$-wide detector slit. A ray would be binned into all the overlapping slots at its point of arrival.

\section{RESULTS AND ANALYSIS}

A drawing of our zero-order filter setup can be seen in Fig. 2. The crucial idea is that the zero order can be filtered out completely by combining a middle stop with a collimating aperture (order-sorting aperture). We use a circular aperture, $40 \mu \mathrm{m}$ in diameter, designed to be slightly smaller than the $50-\mu \mathrm{m}$-diam middle stop. This ensures that the zero-order beam is completely blocked regardless of where the aperture is placed in the beam line as long as the zone plate and aperture are aligned. To ensure maximum focused intensity transfer, the position of the aperture is adjusted so that the cone of the beam coming into focus matches the diameter of the aperture. Because the wavelength of the atoms is so small (less than $0.1 \mathrm{~nm})$ compared to the diameter of the aperture $(20-50 \mu \mathrm{m})$, the optimum position can be found through simple geometrical optics. For our system here, using a Fresnel zone plate with a diameter of $192 \mu \mathrm{m}$ and an image distance of $205 \mathrm{~mm}$, the optimum position of the $40-\mu \mathrm{m}$-diam aperture is $42.7 \mathrm{~mm}$ 


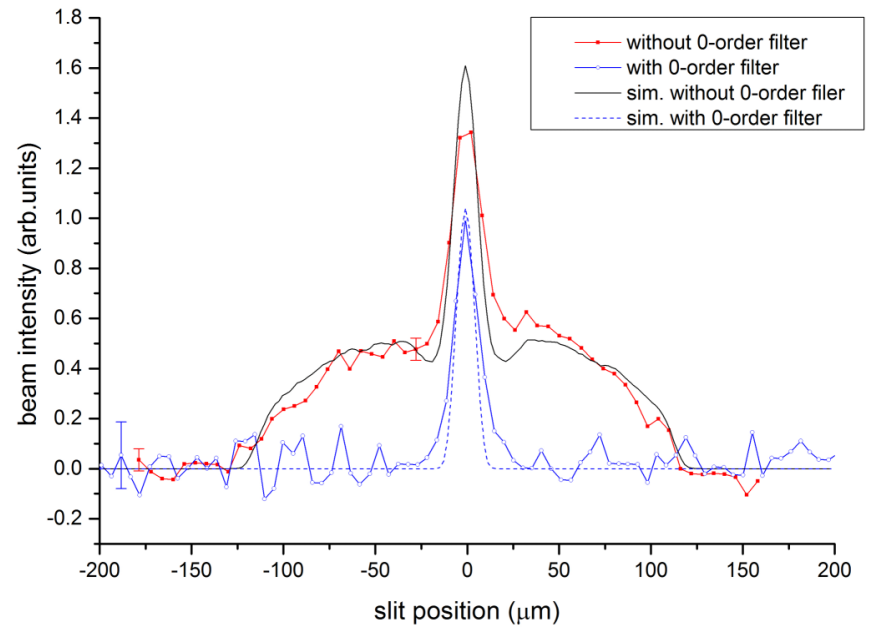

FIG. 3. Experimental focusing results showing two focused beams with and without zero-order filtering together with MCSTAS simulations. The two intensity curves have been normalized relative to the maximum intensity measured with zero-order filtering and the background has been subtracted for both curves. As can be seen, the zero-order background is completely removed by our zero-order filter. These experiments were carried out at a pressure of 76 bars, using a $10-\mu \mathrm{m}$ diameter skimmer.

from the image plane. For our measurements the distance was about $43 \mathrm{~mm}$.

Figure 3 shows experimental results and simulations of focusing of a 76-bar helium beam down to about $3 \mu \mathrm{m}$. The results were obtained using the $10-\mu \mathrm{m}$-diam skimmer. The two graphs in the figure show focusing with and without the zeroorder filter. Zero-order filtering is successfully demonstrated. There is good agreement between simulations and experimental data for both the filtered and unfiltered measurements. The measured intensity in the focus for the unfiltered beam is slightly lower than predicted by the simulations. Further, the footprint to the right is slightly raised compared to the simulations. Both of these effects can be explained as a slow response of the pressure gauge to a sudden pressure change. First, the gauge does not respond rapidly enough to the pressure increase, which leads to a slightly too low maximum intensity measurement. Then it does not respond rapidly enough to the pressure drop, which leads to a raised footprint. The slit was scanned from the left to the right quite fast. Each data point corresponds to about a 5-s measurement time.

Figure 4 shows focusing results for varying pressures from 11 to 81 bars corresponding to speed ratios between 9.0 and 25.8. The results were obtained using the 50- $\mu \mathrm{m}$-diam skimmer. The predictions of the analytical models for speed ratios of 9.0 and 25.8 are plotted convoluted with a $10-\mu \mathrm{m}$ slit function to match the experimental data. MCSTAS simulations for speed ratios 9.0 and 25.8 are also plotted. There is no change in focus size with speed ratio for the experimental data. It can clearly be seen that for the 50- $\mu \mathrm{m}$-diam skimmer used here the analytical model overestimates the focus size for low-speed ratios and does not reproduce the footprint. The reason for this is that the analytical model corresponds to a situation where each point source illuminates uniformly in all directions. The model does not account for the change in

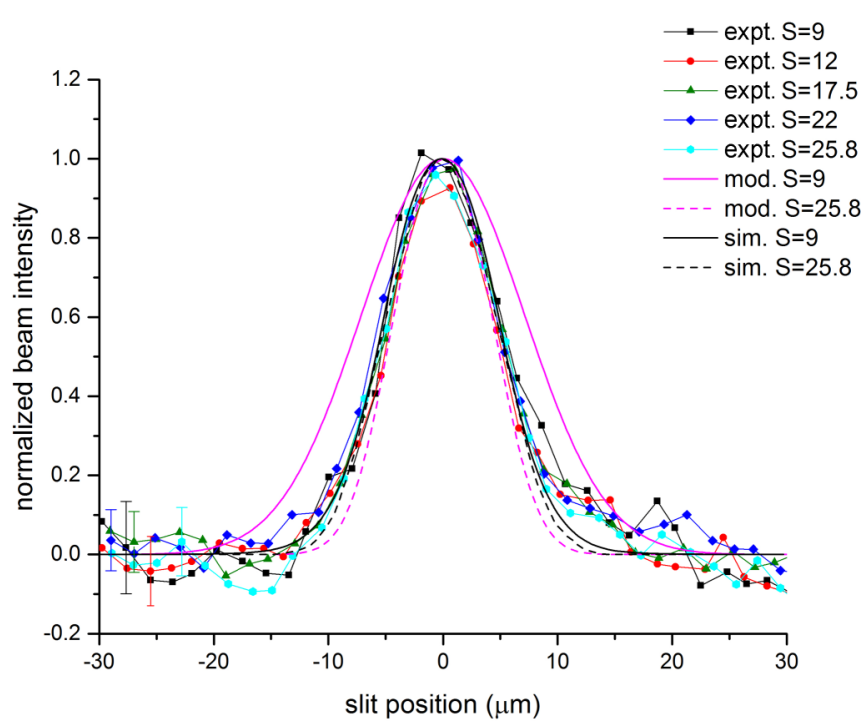

FIG. 4. Experimental focusing results for a 50- $\mu \mathrm{m}$ skimmer with pressures ranging from 11 to 81 bars, plotted together with simulations (sim.) and the analytical model (mod.); here $S$ denotes the speed ratio.

angular distribution of the beam incident on the zone plate for off-axis points on the source. The larger the source (skimmer) and the smaller the speed ratio, the larger this effect will be. It has previously been shown that the analytical model provides good agreement for small skimmers in the micrometer range (see, for example, [9]). The MCSTAS simulations predict only a very small change in focus size with speed ratio and fit the data very well except for the footprint to the right, which is slightly raised. The reason for this is presumably the slow response of the pressure gauge to the pressure drop discussed already for Fig. 3. Further simulations (not shown) show that for skimmer sizes in the micrometer range, the two models converge.

\section{CONCLUSION}

In this paper we presented the experimental realization of a zero-order filter (order-sorting aperture) for diffractive de Broglie matter wave focusing elements. We used the zero-order filter to perform measurements of focusing of a neutral helium beam with source stagnation pressures between 11 and 81 bars. We showed that an analytical model previously used in the literature overestimates the focus size for low-speed ratios for the 50- $\mu \mathrm{m}$-diam skimmer used in our experiments. We attributed this to the fact that the angular distribution of the beam incident on the zone plate changes as the source points move away from the axis. This effect was not accounted for in the analytical model. Simulations of the focusing results using the program package MCSTAS with an ellipsoidal quitting surface source model yielded very good agreement with the experimental results.

\section{ACKNOWLEDGMENT}

We acknowledge funding from the European Union through Theme NMP.2012.1.4-3, Grant No. 309672, Project NEMI. 
[1] A. D. Cronin, J. Schmiedmayer, and D. E. Pritchard, Rev. Mod. Phys. 81, 1051 (2009).

[2] M. Arndt, A. Ekers, W. von Klitzing, and H. Ulbricht, New J. Phys. 14, 125006 (2012).

[3] T. Juffmann, H. Ulbricht, and M. Arndt, Rep. Prog. Phys. 76, 086402 (2013).

[4] D. Farías and K.H. Rieder, Rep. Prog. Phys. 61, 1575 (1998).

[5] G. Bracco and B. Holst, Surface Science Techniques (Springer Science \& Business Media, New York, 2013).

[6] O. Carnal, M. Sigel, T. Sleator, H. Takuma, and J. Mlynek, Phys. Rev. Lett. 67, 3231 (1991).

[7] R. B. Doak, R. E. Grisenti, S. Rehbein, G. Schmahl, J. P. Toennies, and C. Wöll, Phys. Rev. Lett. 83, 4229 (1999).

[8] M. Koch, S. Rehbein, G. Schmahl, T. Reisinger, G. Bracco, W. E. Ernst, and B. Holst, J. Microsc. 229, 1 (2008).

[9] S. D. Eder, T. Reisinger, M. M. Greve, G. Bracco, and B. Holst, New J. Phys. 14, 073014 (2012).

[10] P. Witham and E. Sánchez, Rev. Sci. Instrum. 82, 103705 (2011).

[11] P. J. Witham and E. J. Sánches, J. Microsc. 248, 223 (2012).

[12] A. Fahy, M. Barr, J. Martens, and P. Dastoor, Rev. Sci. Instrum. 86, 023704 (2015).

[13] M. Barr, A. Fahy, J. Martens, A. Jardine, D. Ward, J. Ellis, W. Allison, and P. Dastoor, Nat. Commun. 7, 10189 (2016).

[14] T. Reisinger and B. Holst, J. Vac. Sci. Technol. B 26, 2374 (2008).

[15] T. Reisinger, G. Bracco, S. Rehbein, G. Schmahl, W. E. Ernst, and B. Holst, J. Phys. Chem. A 111, 12620 (2007).

[16] S. Eder, G. Bracco, T. Kaltenbacher, and B. Holst, J. Phys. Chem. A 118, 4 (2013).

[17] T. Reisinger, G. Bracco, and B. Holst, New J. Phys. 13, 065016 (2011).

[18] T. Judd, R. Scott, G. Sinuco, T. Montgomery, A. Martin, P. Krüger, and T. Fromhold, New J. Phys. 12, 063033 (2010).

[19] S. D. Eder, X. Guo, T. Kaltenbacher, M. M. Greve, M. Kalläne, L. Kipp, and B. Holst, Phys. Rev. A 91, 043608 (2015).
[20] L. Kipp, M. Skibowski, R. Johnson, R. Berndt, R. Adelung, S. Harm, and R. Seemann, Nature (London) 414, 184 (2001).

[21] R. D. Doak, in Helium Atom Scattering from Surfaces, edited by E. Hulpke (Springer, Berlin, 1992), Vol. 27.

[22] B. Holst and W. Allison, Nature (London) 390, 244 (1997).

[23] K. Fladischer, H. Reingruber, T. Reisinger, V. Mayrhofer, W. E. Ernst, A. E. Ross, D. A. MacLaren, W. Allison, D. Litwin, J. Galas, S. Sitarek, P. Nieto, D. Barredo, D. Farías, R. Miranda, B. Surma, A. Miros, B. Piatkowski, E. Søndergård, and B. Holst, New J. Phys. 12, 033018 (2010).

[24] P. Sutter, M. Minniti, P. Albrecht, D. Farías, R. Miranda, and E. Sutter, Appl. Phys. Lett. 99, 211907 (2011).

[25] H. C. Schewe, B. S. Zhao, G. Meijer, and W. Schöllkopf, New J. Phys. 11, 113030 (2009).

[26] M. M. Greve, A. M. Vial, J. J. Stamnes, and B. Holst, Opt. Express 21, 28483 (2013).

[27] A. Sakdinawat and D. Attwood, Nat. Photon. 4, 840 (2010).

[28] K. Lefmann and K. Nielsen, Neutron News 10, 20 (1999).

[29] P. Willendrup, E. Farhi, and K. Lefmann, Physica B 350, E735 (2004)

[30] S. Eder, B. Samelin, G. Bracco, K. Ansperger, and B. Holst, Rev. Sci. Instrum. 84, 093303 (2013).

[31] T. Reisinger, S. Eder, M. Greve, H. Smith, and B. Holst, Microelectron. Eng. 87, 1011 (2010).

[32] H. Pauly, in Atom, Molecule, and Cluster Beams I, edited by H. Pauly (Springer, Berlin, 2000), Vol. 1.

[33] J. Toennies and K. Winkelmann, J. Chem. Phys. 66, 3965 (1977).

[34] L. Pedemonte and G. Bracco, J. Chem. Phys. 119, 1433 (2003).

[35] A. G. Michette, in Optical Systems for Soft X Rays, edited by A. Michette (Plenum, New York, 1968).

[36] MCSTAS, a ray-tracing simulation software package, available at www.mcstas.org.

[37] H. C. W. Beijernick and N. F. Verster, Physica C 111, 327 (1981).

[38] A. S. Palau, G. Bracco, and B. Holst, Phys. Rev. A 94, 063624 (2016). 